\title{
BPM System Improvements
}

\author{
M. Church
}

24 April 1991

\section{Motivation}

During the accelerator studies period of 12/90 through 1/91 the Accumulator BPM system was investigated in some detail in an effort to improve its reliability and accuracy in making closed orbit measurements. The motivation for this is to try and improve the beam energy resolution for $\mathrm{E} 760$. The relativistic $\beta$ of the $\bar{p}$ is given by

$$
\beta=f_{R} L / c
$$

where $f_{R}$ is the revolution frequency, $L$ is the orbit length $(\sim 474050 \mathrm{~mm})$, and $c$ is the speed of light. Hence, the error in $\beta$ is given by

$$
d \beta / \beta=d f_{R} / f_{R}+d L / L
$$

Since $d f_{R} / f_{R}$ is $\sim 2 \times 10^{-7}$, the main contribution to the error comes from $d L$. During the E760 run of $5 / 90$ to $9 / 90 d L$ was estimated to be $\sim 1 \mathrm{~mm}$. It is thought that this can be reduced to $\sim .25 \mathrm{~mm}$ with proper use of the present BPM system.

$L$ is given by

$$
L=L_{0}+\delta L
$$

where $L_{0}$ is the accurately known orbit length of a reference orbit (extracted from an energy scan of the $J / \Psi$ or $\Psi^{\prime}$ ), and $\delta L$ is the difference orbit between the current orbit and the reference orbit. $\delta L$ is calculated in the 1st approximation by

$$
\delta L=\sum_{i} C_{i} \sum_{j} \Delta B P M_{i j}
$$

where $\triangle B P M_{i j}$ is the horizontal difference orbit at the $i$ th BPM in the $j$ th sector and $C_{i}$ are constants depending upon the location of the BPM pickup and the strength of the quadrupoles. ${ }^{1}$ Table I lists the constants $C_{i}$, and Fig. 1 shows a typical difference orbit, $\triangle B P M_{i j}$.

These studies were all done with "reverse protons" and concentrated on closed orbit measurements with the Accumulator horizontal BPMs. The low frequency $(\mathrm{H}=2)$ mode of the BPM system is used in all cases, therefore it is required that the beam be bunched with ARF3 at some level. ${ }^{2}$ The low frequency RF module in the BPM

\footnotetext{
${ }^{1}$ For a more accurate calculation of the orbit length difference, see $\mathbf{S}$ Werkema, future Pbar Note.

${ }^{2}$ See FNAL TM-1254, Bagwell, et al, 1984, for a fairly complete, although slightly out-of-date description of the Antiproton Source Beam Position System.
} 
system had previously been modified to track the $\mathrm{H}=2$ frequency

\section{Sources of BPM Error}

There are 4 potential sources of errors in the BPM system that have been identified and corrected: 1) miscalibration of the BPM gain; 2) finite size of the LSB (least significant bit) of the $\mathrm{ADC} ; 3$ ) system nonlinear behaviour; and 4) incorrect electrical offsets.

\section{A. BPM Calibration}

In principle, the calibration of a particular BPM (ratio between beam position and ADC counts) depends only on the geometry of the pickup. However due to phase mismatches and other imperfections in the electronics, the calibration also depends on the particular amplifiers used, and therefore each individual BPM must be separately calibrated to obtain submillimeter accuracy. The absolute calibration of a high dispersion BPM (@Q10, Q11, Q14) was obtained by using the 4-8 Ghz momentum cooling pickup located at A20. The beam was bunched and moved in increments of several millimeters by varying the $R F$ frequency. At each step the 4-8 pickup was moved such that the characteristic notch signal was symmetric (see Fig. 2), thus insuring that the beam was centered under the pickup. Even though the 4-8 pickup is directional, a strong notch signal is evident due to an impedance mismatch in the pickup signal leads. ${ }^{3}$ Fig. 3 shows a plot of BPM position vs. $4-8$ pickup position. The 4-8 pickup itself is calibrated with a dial indicator mounted on the cooling tank.

An absolute calibration of a low dispersion BPM (@Q1, Q3, Q4, Q6, Q8) was obtained using the accurately calibrated scraper A:RJ500 at A50. Fresh beam was placed at different horizontal locations at A50 (using a local bump), and its position measured with the BPMs at A5Q1 and A4Q1. A scraper scan with RJ500 was then performed and the extinction point measured. Fig. 4 shows a plot of BPM position vs. extinction point.

The relative calibration of the remaining BPMs was obtained by inputting a differential test signal to each BPM and recording the resultant output position. Since the magnitude of the test signal varies from BPM to BPM, this had to be measured also (see Table II). The absolute calibration of a single BPM and the relative calibration of each BPM are combined to give an absolute calibration of each BPM. These numbers are entered as constants in P76 (E760 Closed Orbits). Fig. 5 shows the percentage change from the original calibration constants for each of the 48 Accumulator horizontal BPMs. Note that A1Q4, A1Q8, A2Q4, and A2Q8 are of a different geometry and have not been recalibrated by this method. The calibration was only done for preamp attenuation $=46 \mathrm{~dB}$ and amplifier gain $=20 \mathrm{~dB}$.

A partial check on the accuracy of the new calibration constants was made by measuring the dispersion at the low dispersion and high dispersion straight sections using

$$
D=\eta(\Delta x) f_{R} / d f_{R}
$$

where $\eta$ is known, $\Delta x$ is measured with the BPMs and $d f_{R} / f_{R}$ is the relative change in RF frequency. The dispersion measured this way should be consistent from sector

\footnotetext{
${ }^{3}$ Private communication, D McGinnis.
} 
to sector and close to the design values. This is in fact the case (see Table III).

\section{B. ADC Sensitivity}

The final BPM signal is digitized by an 8 bit ADC which has 3 different sensitivity settings-4, 2, and 1 . (This is actually just a programmable attenuator in the BPM Gain and Offset Module.) Traditionally, only sensitivity $=1$ had been used, which yields an ADC LSB of $1.2 \mathrm{~mm}$ for the high dispersion BPMs and $.33 \mathrm{~mm}$ for the low dispersion BPMs. The sensitivity $=4$ setting was tested and found to give valid position measurements, although it requires a different set of electrical offsets than sensitivity $=1$. The LSB is thus reduced to $.3 \mathrm{~mm}$ for the high dispersion BPMs and $.08 \mathrm{~mm}$ for the low dispersion BPMs. However, this sensitivity setting limits the full range of BPM measurements to $\sim \pm 40 \mathrm{~mm}$, which does not cover the full aperture of the Accumulator.

\section{Nonlinear Behaviour}

The amplitude of the signal from the BPM pickup depends on 3 parameters - the beam current, the RF voltage, and the frequency spread of the beam. Attenuation settings of 0 and $46 \mathrm{~dB}$ for the preamplifier, and gain settings of $0,20,40$, and 60 $\mathrm{dB}$ for the amplifier permit, in principle, a dynamic range of $106 \mathrm{~dB}$. However, it was found that the $60 \mathrm{~dB}$ gain setting for the amplifier (actually a $20 \mathrm{~dB}$ and 40 $\mathrm{dB}$ cascaded pair) yields very unreliable position measurements, and that the $40 \mathrm{~dB}$ gain setting yields position measurements with poor accuracy. Furthermore, for the settings, preamp $=46 \mathrm{~dB}$ attenuation and $\mathrm{amp}=0 \mathrm{~dB}$ gain, the test signal is not large enough to generate reliable electrical offsets. There remains only 3 reliable overall gain settings:

preamp $=46 \mathrm{~dB}$ atten, amp $=20 \mathrm{~dB}$ gain;

preamp $=0 \mathrm{~dB}$ atten, $\mathrm{amp}=0 \mathrm{~dB}$ gain;

preamp $=0 \mathrm{~dB}$ atten, $a m p=20 \mathrm{~dB}$ gain.

In each configuration there is a linear dynamic range of about $20 \mathrm{~dB}$ in amplitude, giving a total dynamic range of $\sim 66 \mathrm{~dB}$. Varying the RF voltage effectively increases this dynamic range. We have been able to measure accurate (repeatable) closed orbits with as little as $.05 \mathrm{~mA}$ of beam

As an aid to finding the most appropriate gain and attenuation settings, an Application Program (P57) has been written which "plateaus" the BPMs as a function of $R F$ voltage: the $R F$ voltage is varied over a given range and BPM measurements are taken and displayed as a function of RF voltage. Fig. 6 shows these plateaus for a suitable set of gain and attenuation, and Fig. 7 shows plateaus for a less suitable choice of gain and attenuation.

\section{Electrical Offsets}

It was found that different electrical offsets are required for different gain and attenuation settings. In addition, a different test signal is required for generating electrical offsets for different configurations. A new Accumulator Closed Orbit program (P76) has been written which allows the user to create, access, and save multiple offset files for different beam conditions. Electrical offsets cannot be accurately gen- 
erated while there is beam in the machine because beam Schottky noise produces a finite BPM signal even when the beam is not bunched. (In fact, with $60 \mathrm{~mA}$ in the machine, fairly good (repeatable) closed orbits measurements may be made with the beam unbunched.)

\section{New Procedures}

A complete set of procedures for taking closed orbits in the Debuncher and Accumulator has been written and placed in the AP10 Accumulator Operations Manual and Debuncher Operations Manual. Procedures are described for testing the BPM system, generating electrical offsets, changing parameters, and taking closed orbits correctly. Hopefully, this will eliminate most of the past confusion in taking closed orbit measurements. 


\begin{tabular}{|c|c|}
\hline BPM & $C$ \\
\hline$Q 1$ & 0.000 \\
\hline$Q 3$ & 0.069 \\
\hline$Q 4$ & 0.019 \\
\hline$Q 6$ & 0.000 \\
\hline$Q 8$ & 0.291 \\
\hline$Q 10$ & 0.614 \\
\hline$Q 11$ & -0.088 \\
\hline$Q 14$ & 0.000 \\
\hline
\end{tabular}

TABLE I: Constants for calculating orbit length from BPM measurements 


\begin{tabular}{|c|r|r|r|r|r|r|}
\hline & 10 & 20 & 30 & 40 & 50 & 60 \\
\hline Q1 & 620 & 622 & 646 & 670 & 618 & 642 \\
\hline Q3 & 582 & 592 & 608 & 632 & 582 & 608 \\
\hline Q4 & 562 & 566 & 582 & 604 & 560 & 580 \\
\hline Q6 & 528 & 532 & 552 & 566 & 524 & 546 \\
\hline Q8 & 578 & 590 & 638 & 650 & 584 & 612 \\
\hline Q10 & 548 & 550 & 590 & 604 & 548 & 580 \\
\hline Q11 & 534 & 536 & 570 & 582 & 530 & 558 \\
\hline Q14 & 522 & 516 & 556 & 556 & 512 & 532 \\
\hline
\end{tabular}

TABLE II: Test signal peak-to-peak amplitude (in $\mathrm{mV}$ ) at input to preamp for differential test $\$ 91$ 
PBEAM $=8834 \mathrm{Mev} / \mathrm{c} \quad \mathrm{ETA}=-0.0236 \quad \mathrm{DP} / \mathrm{P}=-0.00408 \quad \mathrm{dF}=.00509 \mathrm{~Hz}$

\begin{tabular}{|c|c|c|c|c|c|c|c|}
\hline sector & BPM & $\begin{array}{c}\text { DX } \\
\text { calib. }\end{array}$ & $\begin{array}{l}\text { DXave } \\
\text { calib. }\end{array}$ & $\begin{array}{l}\text { DISP (m) } \\
\text { calib. }\end{array}$ & $\begin{array}{c}\text { DX } \\
\text { uncal ib }\end{array}$ & $\begin{array}{c}\text { DXave } \\
\text { uncal ib. }\end{array}$ & $\begin{array}{c}\text { DISP (m) } \\
\text { uncal ib. }\end{array}$ \\
\hline$\overline{\mathrm{A} 1}$ & $\begin{array}{l}\text { A6Q1 } \\
\text { A1Q1 }\end{array}$ & $\begin{array}{l}-1.19 \\
-0.34\end{array}$ & -0.77 & 0.19 & $\begin{array}{l}-1.00 \\
-0.33\end{array}$ & -0.67 & 0.17 \\
\hline A2 & $\begin{array}{l}\text { A1Q14 } \\
\text { A2Q14 }\end{array}$ & $\begin{array}{l}-44.02 \\
-34.05\end{array}$ & -39.03 & 9.56 & $\begin{array}{l}-50.02 \\
-44.80\end{array}$ & -47.41 & 11.61 \\
\hline A3 & $\begin{array}{l}\text { A2Q1 } \\
\text { A3Q1 }\end{array}$ & $\begin{array}{l}-0.72 \\
-1.16\end{array}$ & -0.94 & 0.23 & $\begin{array}{l}-0.67 \\
-1.01\end{array}$ & -0.84 & 0.21 \\
\hline A4 & $\begin{array}{l}\text { A3Q14 } \\
\text { A4Q14 }\end{array}$ & $\begin{array}{l}-39.19 \\
-38.27\end{array}$ & -38.73 & 9.49 & $\begin{array}{l}-42.60 \\
-43.00\end{array}$ & -42.80 & 10.49 \\
\hline A5 & $\begin{array}{l}\text { A4Q1 } \\
\text { A5Q1 }\end{array}$ & $\begin{array}{l}-1.23 \\
-1.05\end{array}$ & -1.14 & 0.28 & $\begin{array}{l}-1.00 \\
-1.00\end{array}$ & -1.00 & 0.25 \\
\hline A6 & $\begin{array}{l}\text { A5Q14 } \\
\text { A6Q14 }\end{array}$ & $\begin{array}{l}-43.52 \\
-34.58\end{array}$ & -39.05 & 9.57 & $\begin{array}{l}-46.30 \\
-39.30\end{array}$ & -42.80 & 10.49 \\
\hline \multicolumn{2}{|c|}{ AVERAGE } & & $\begin{array}{l}\text { low disp } \\
\text { igh disp }\end{array}$ & $\begin{array}{l}0.23 \\
9.54\end{array}$ & & & $\begin{array}{r}0.21 \\
10.86\end{array}$ \\
\hline
\end{tabular}

TABLE III: Dispersion measurement with calibrated and uncalibrated BPMs 

ACEUMULATOR CLDSEII DREIT
A:RILLFS
52.792
4.57

CO Date/Time: 12/18/90 0653:52

AVG OYER 20 FRAMES ( $\begin{gathered}20 \\ 20 \\ \text { Deltap } / p=0\end{gathered}$

\section{BEAM POSITIONS 〈mm}

\begin{tabular}{|ll}
$A 1 Q 1$ & -1 \\
$A 1 Q 3$ & 0 \\
A1Q4 & -.5 \\
A1Q6 & -1.34 \\
A1Q8 & 2.47 \\
A1Q10 & -1.21 \\
A1Q11 & -2.41 \\
A1Q14 & -2.41
\end{tabular}

\begin{tabular}{lc|ll}
$A 2 Q 14$ & 1.21 & $A 3 Q 1$ & .67 \\
$A 2 Q 11$ & 2.41 & $A 3 Q 3$ & $i$ \\
$A 2 Q 10$ & 2.41 & $A 3 Q 4$ & -.33 \\
$A 2 Q 8$ & -1.5 & $A 3 Q 6$ & -3.67 \\
$A 2 Q 6$ & 3.99 & $A 3 Q 8$ & -4.65 \\
$A 2 Q 4$ & 3.53 & $A 3 Q 10$ & 1.21 \\
$A 2 Q 3$ & -.33 & $A 3 Q 11$ & 1.21 \\
$A 2 Q 1$ & 0 & $A 3 Q 14$ & 1.21
\end{tabular}

$\begin{array}{|lc|}A 4 Q 14 & -2.4 \\ A 4 Q 11 & -2.41 \\ \text { A4Q10 } & -1.2 \\ \text { A4Q8 } & 1.69 \\ \text { A4Q6 } & 1.99 \\ \text { A4Q4 } & .33 \\ \text { A4Q3 } & -.66 \\ \text { A4Q1 } & -1\end{array}$

$A 5 Q 1-2.01$

$\begin{array}{lll}A 5 Q 3 & -2.33\end{array}$

$\begin{array}{ll}A 5 Q 4 & -1.32\end{array}$

A5Q6 1.33

A5Q8 4.87

ASQ10 -2.4

A5Q11 -2.41

A5Q14 -1.2

\begin{tabular}{|ll}
$A 6 Q 14$ & 1.21 \\
$A G Q 11$ & 1.21 \\
$A 6 Q 10$ & 0 \\
$A 6 Q 8$ & -2 \\
$A 6 Q 6$ & 2.33 \\
$A 6 Q 4$ & -2.67 \\
$A G Q 3$ & -3.99 \\
$A 6 Q 1$ & -3.34
\end{tabular}






CONSOLE 15, 12-DEC-1999 17:58

- $\quad$ Thar sa flot

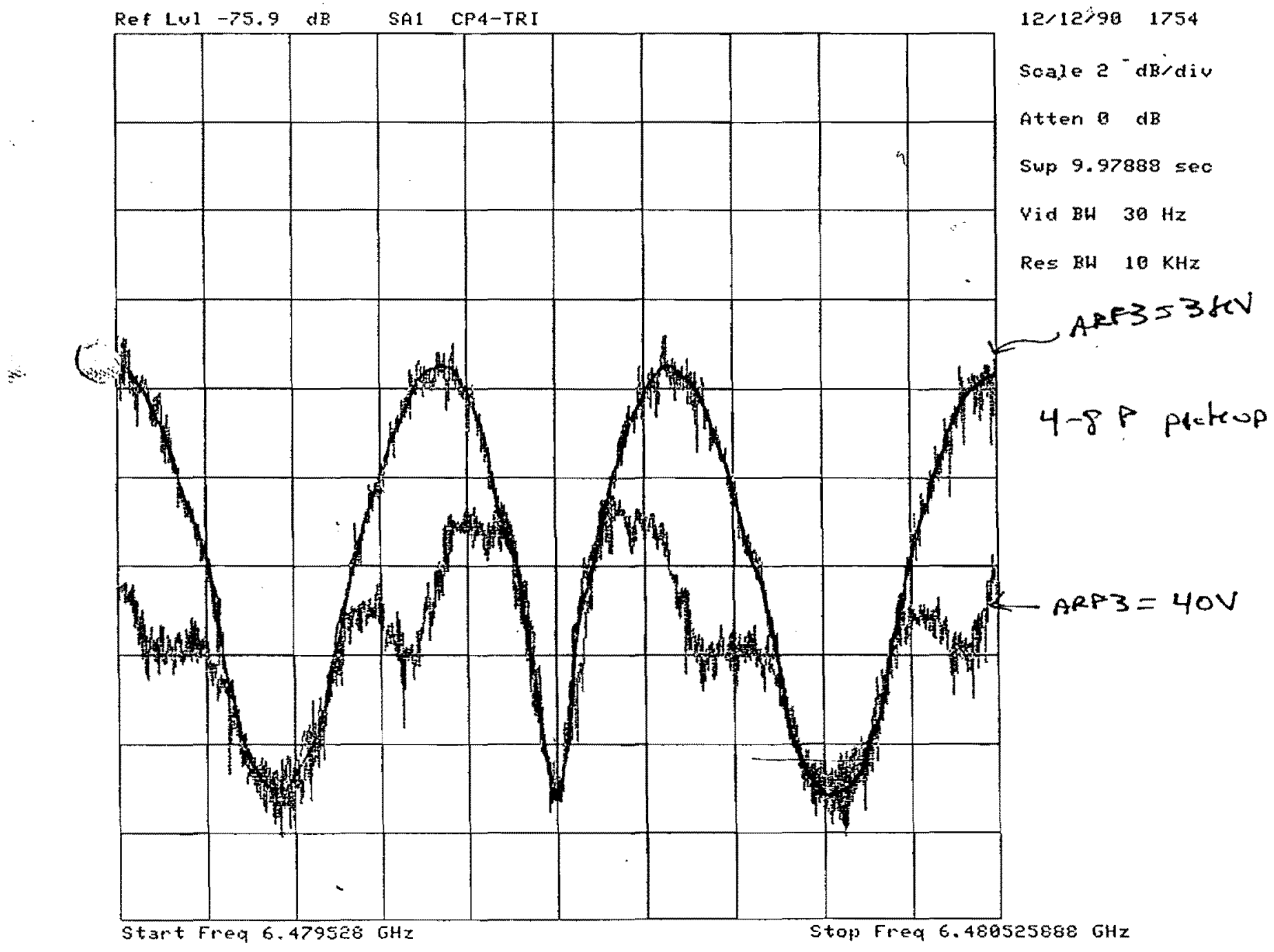

(-...

FIG. 2: Notch signal from 4-8 momentum stochastic cooling pickup; bunched and unbunched beam, reverse protons 
AMP GAIN $=0$

ATTEN $=\varnothing$

Polynomial Curve Fit

ADE GAIN $=1$

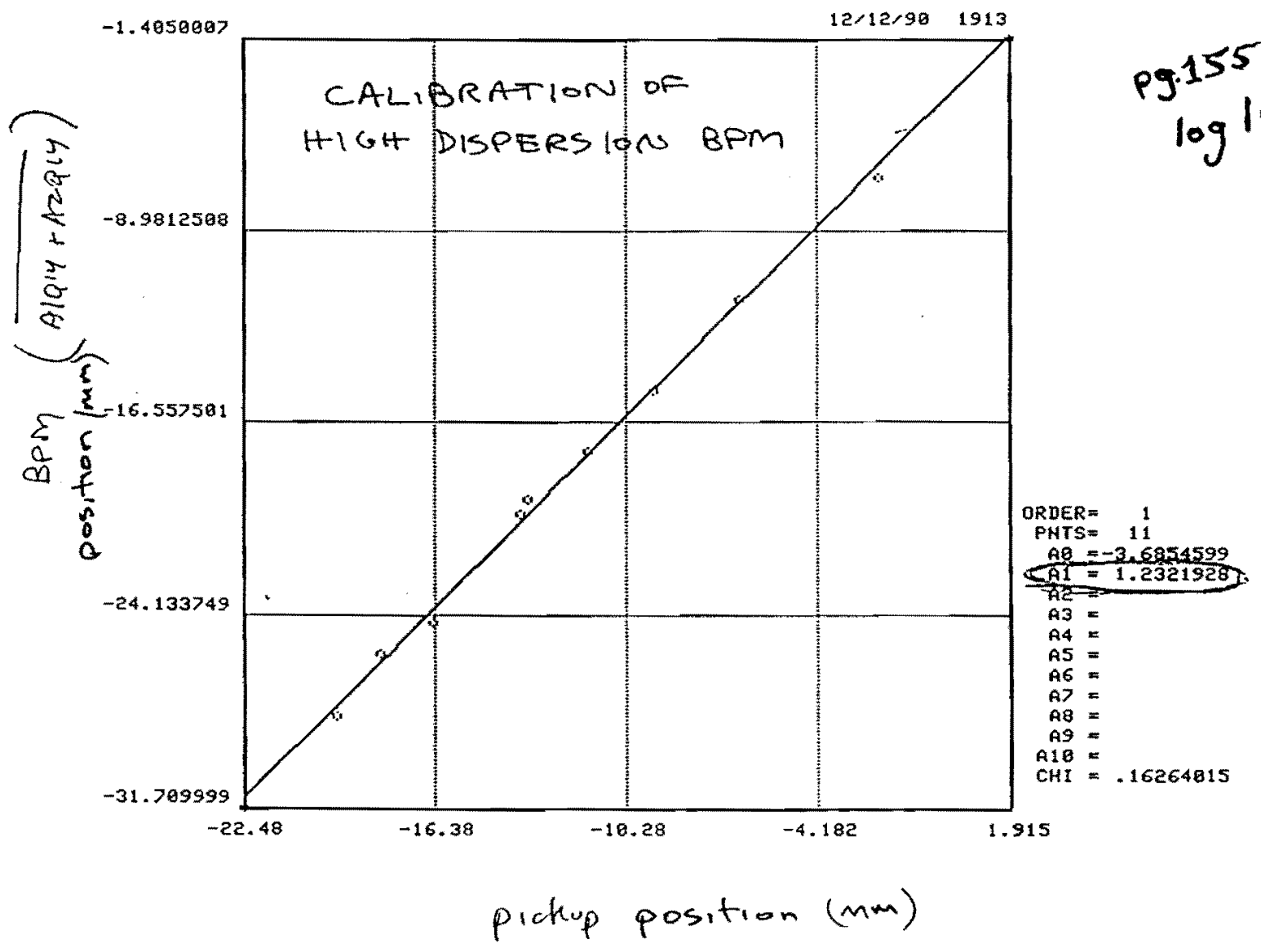

FIG. 3: Calibration of high dispersion BPM with 4-8 momentum cooling pickup 


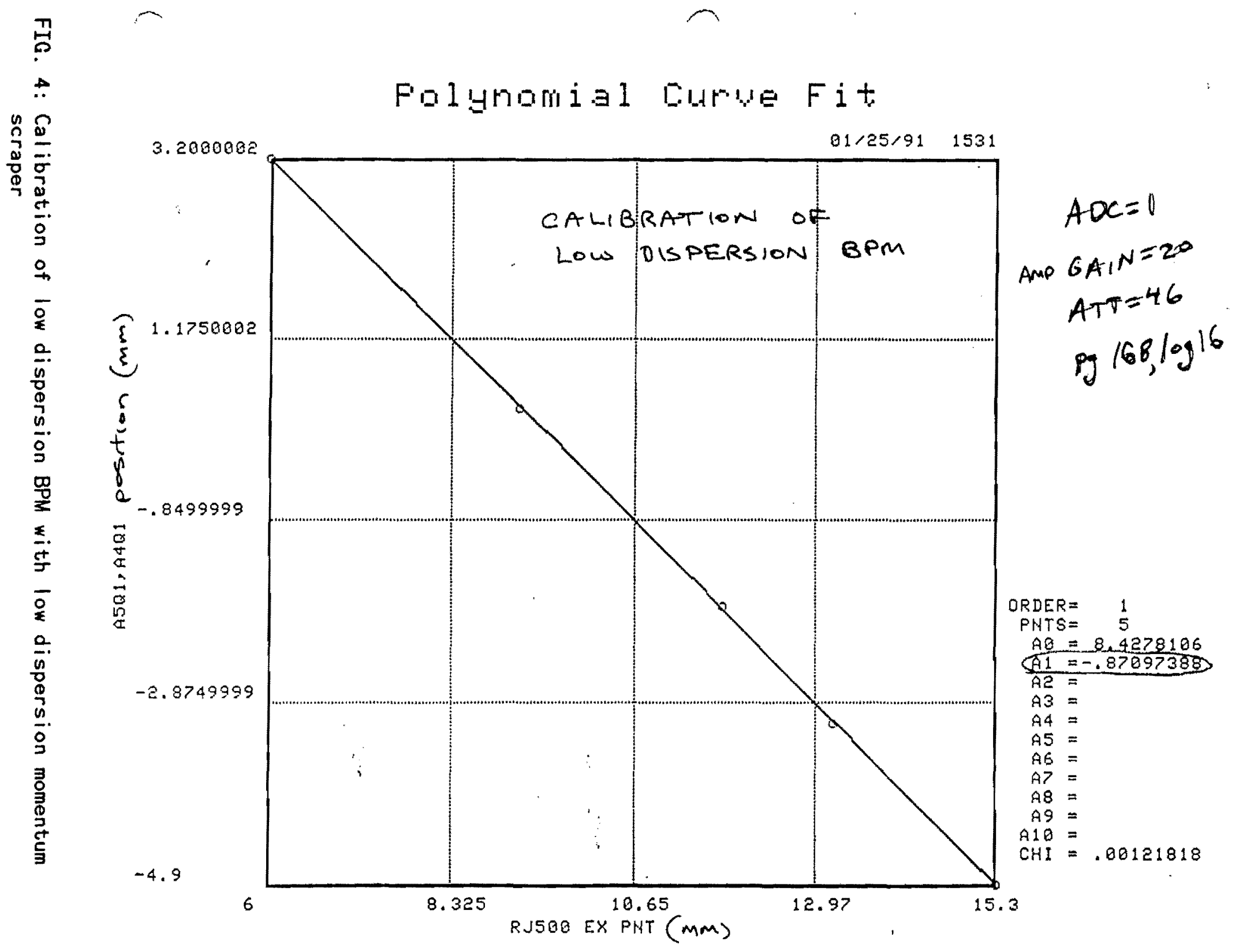


ACCUMULATOR HORIZONTAL


$-30$

FIG. 5: \% change between old BPM calibration and new BPM calibration 
:

$\therefore \quad$ CONSOLE LOCATION 08, AF10 S-JAN-1991 14:00 $8 \mathrm{~mA}$ beam ADCATEAUS

$A+\tan =46 \quad$ Garn $=20$ BPM PLATEAUS
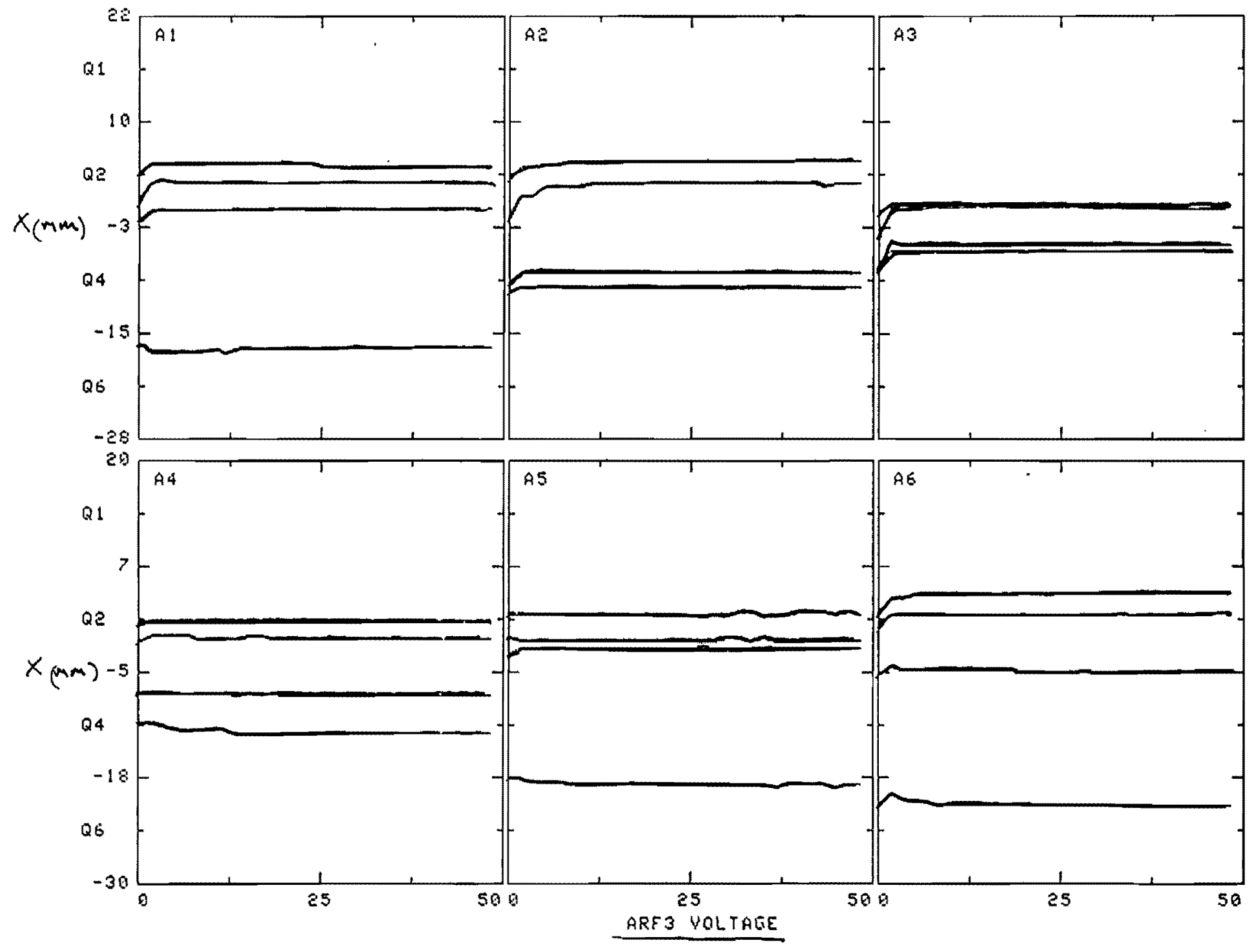




